\title{
Dynamic Designs of 3D Virtual Worlds Using Generative Design Agents
}

\author{
GU Ning and MAHER Mary Lou \\ Key Centre of Design Computing and Cognition, University of Sydney
}

Keywords: Virtual Environments, Generative Design, Interactive Design, Shape Grammars.

Abstract: 3D virtual worlds are networked environments designed using the metaphor of architecture. Recent developments in 3D virtual worlds focus on interactivity, flexibility and adaptability. Rather than creating virtual environments in which the objects have intelligent behaviours, our research takes a different approach to develop an agent model that is associated with an individual person in the $3 \mathrm{D}$ virtual world as a personal design agent. This paper presents a Generative Design Agent (GDA), a kind of rational agent capable of representing a person in a virtual world and designing, implementing and demolishing 3D virtual places based on the occupants' current needs in the virtual world. The core of a GDA's design component is a generative design grammar that is able to capture a style of 3D virtual worlds. 3D virtual worlds designed using the GDA model is another kind of architecture for the "moment".

\section{INTRODUCTION: 3D VIRTUAL WORLDS}

Virtual worlds, virtual architecture or cyberspace can be understood as networked environments designed using the metaphor of architecture. Through the use of metaphor, we express the concepts in one domain in terms of another (Lakoff and Johnson 1980). The architectural metaphor provides a consistent context for people to browse digital information, interact with the environment and communicate with each other. The purposes of 3D virtual worlds have expanded from the original internet gaming and military simulation to provide support for other activities such as online learning and research, virtual design studios, virtual museums and so on. Virtual worlds have the potential to develop alongside the built environments to be an essential part of our living environments.

Designing virtual worlds has accommodated many different technologies supporting multi-user text-based, 2D graphical and 3D virtual worlds. Nowadays, 3D virtual worlds have become the most common forms. These worlds use 3D models for representing places. A person appears as an avatar (an animated character) which locates the view of the world and provides a sense of awareness of others in the world. Examples of 3D virtual worlds are among those designs implemented with platforms like Active Worlds (www.activeworlds.com), Adobe Atmosphere (www.adobe.com/atmosphere), Virtools (www.virtools.com), and SecondLife 
(www.secondlife.com). Figure 1 illustrates selected designs in 3D virtual worlds from our recent research and teaching.

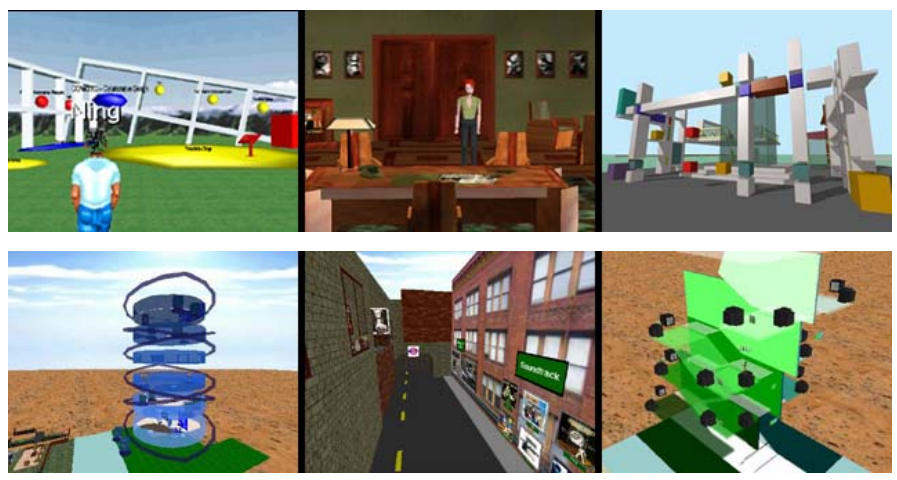

Figure 1 Designs in the 3D Virtual Worlds

An analogy between 3D virtual worlds and the built environments is based on the view that $3 \mathrm{D}$ virtual worlds are comprised of functional places. The designer specifies and organises meaningful places and objects, reflecting some social and cultural needs and values in a particular form. Such a view assumes that the concepts of $3 \mathrm{D}$ virtual worlds and the built environments have some commonalities as well as some relevant differences. Except for the input and output devices, 3D virtual worlds are implemented entirely in a computer environment. Therefore, the worlds are basically assemblies of computing entities, which can be flexibly programmed and configured. This flexibility makes it possible to consider designing 3D virtual worlds in terms of dynamics and autonomy. However, current examples of $3 \mathrm{D}$ virtual worlds are largely static. Like the built environments, the design process is separated in time from its use, and therefore is not directly related to the individual's experience of these virtual places. This paper presents a Generative Design Agent (GDA) model for dynamic designs of 3D virtual worlds. A GDA is a kind of rational agent, on one hand represents a person in the virtual world capable of providing agencies for interacting with other GDAs and the environment, and on the other hand acts as a design agent capable of designing, implementing and demolishing 3D virtual places as needed. The core of a GDA's design component is a generative design grammar that is able to capture a style of $3 \mathrm{D}$ virtual worlds.

\section{AGENT MODELS FOR 3D VIRTUAL WORLDS}

An increased interest in computational agents has been seen recently in the development of internet applications influenced by the concepts of artificial intelligent and artificial life. In the context of computer science, agents as intentional systems operate independently and rationally, seeking to achieve goals by interacting with their environment (Wooldridge and Jennings 1995). Unlike most computational objects, agents have goals and beliefs and execute actions based on 
these goals and beliefs (Russell and Norvig, 1995). The increasing interconnection and networking of computers requires agents to interact with each other (Huhns and Stephens 1999). The concept of a multi-agent system is therefore introduced with the applications of distributed artificial intelligence.

Examples such as internet gaming environments usually associate pre-programmed behaviors with $3 \mathrm{D}$ objects to integrate interactions to $3 \mathrm{D}$ virtual worlds. This allows some components of the environment to be interactive by performing a fixed set of responses. Instead of ascribing fixed behaviours to 3D objects in a virtual world, Maher and Gero (2002) propose a multi-agent system (Figure 2) to represent a 3D virtual world so that each object in the world has agency. With sensors and effectors as the interface to the 3D virtual world, each agent can sense the world, reason about the goals and modify the virtual world to satisfy the goals. 3D virtual worlds developed using this model can adapt their designs to suit different needs.

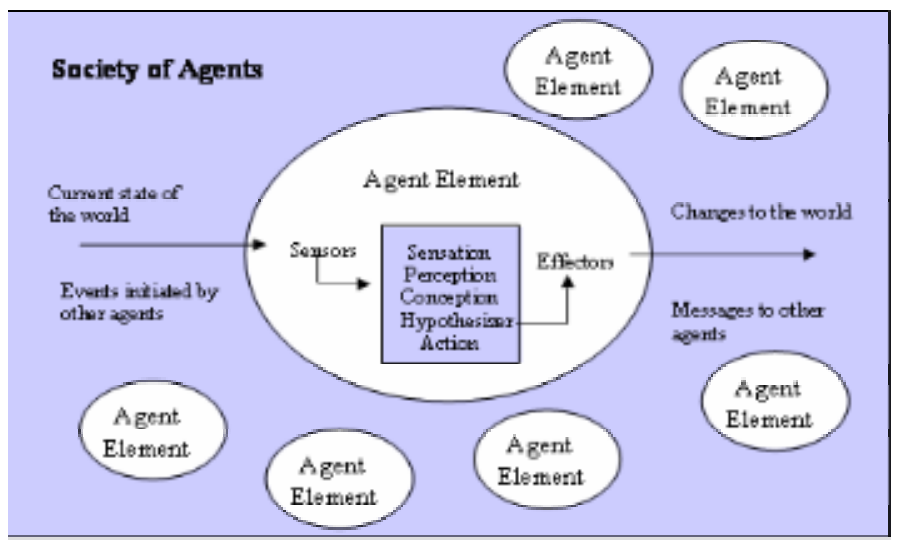

Figure 2 A 3D Virtual World as a Multi-agent System (Maher and Gero 2002)

The typical application of this multi-agent system is to develop object agents in 3D virtual worlds by applying agencies to structural components of a $3 \mathrm{D}$ virtual world such as wall, floor and so on, or to spatial components of the world such as room, building and so on. With the specification of relevant domain knowledge, this model has been applied to dynamically control information flows in designing 3D models and to increase the level of interactivity in 3D virtual worlds (Maher et al. 2003).

\section{GENERATIVE DESIGN AGENT MODEL}

Our study takes a different approach by developing augmented design agents rather than object agent, for 3D virtual worlds. Therefore, the Generative Design Agent (GDA) model is proposed. On one hand, a GDA represents a person in the virtual world by providing this individual with agencies for interacting with other GDAs and the environment. On the other hand, it serves as a design agent that can design, implement and demolish 3D virtual places as needed. The core of the design 


\section{Dynamic Designs of 3D Virtual Worlds Using Generative Design Agents}

component is a generative design grammar that captures a style of 3D virtual worlds, which can be applied to designing virtual places in 3D virtual worlds as needed. Using the GDA model, a multi-user virtual world can be represented by a society of GDAs. Each GDA corresponds to an individual of the virtual community.

As the representation of a person in the virtual world, a GDA is able to interact with other GDAs and the environment. By monitoring these activities, the GDA interprets the person's current needs and automatically designs 3D virtual places to satisfy these needs. With the GDA model, the most significant change to a 3D virtual world is that the design of the world does not need to be pre-defined like the built environment. The 3D virtual world is designed, implemented and demolished, to suit the needs of its inhabitants. The GDA model also allows the design of the world to have influence from its inhabitants, rather than being solely controlled by the designers. The following highlights the characteristics of the GDA approach.

- The agency is applied to a person in the virtual world instead of each structural or spatial component of the environment. Each GDA is a personal design agent.

- The GDA interacts with other GDAs and the environment. By observing these activities, the current needs of the person and his/her collaborators are interpreted.

- Designers do not specify every design. Instead, they specify design grammars for the GDAs. The application of the design grammar then is directed by the currently interpreted needs of the inhabitants, to generate the actual designs of $3 \mathrm{D}$ virtual places in the world, for the moment.

The design process can be modeled as the interaction of the three worlds: the external world, the interpreted world and the expected world (Gero, 2002).

- The external world is the world that is composed of external representations of the design outside the designers.

- The interpreted world is the internal representation of the external world that exists inside the designer in terms of the designer's knowledge, experiences and interpretations of the external world.

- The expected world is the world in which the effects of design decisions are predicted based on the designer's current design goals and his/her interpretations of the current state of the world.

The computational processes of the GDA common model are proposed on the base of Maher and Gero's agent model for 3D virtual worlds (2002), where sensors and effectors act as the interface between the agent and the $3 \mathrm{D}$ virtual world. There are three computational processes: interpretation, hypothesising and designing. The external world, the GDA's interpreted world and expected world are connected via these three computational processes (Figure 3).

- In interpretation, the GDA uses sensor(s) to retrieve raw data from the 3D virtual world (external world). These data are filtered, focused and transformed to construct the interpreted world, where the current needs of the inhabitants and the current state of the world are interpreted. The types of data from the external world are avatars (A), events (E), attributes of the virtual world such as system time, number of users, object path of the world (W) and 3D objects $(\mathrm{O})$. 
- In hypothesising, the GDA constructs the expected world by setting up design goals to reduce or eliminate the mismatches between the currently interpreted needs of the occupants and the state of the world.

- In designing, the GDA applies a design grammar to provide design solutions for satisfying the design goals. The design solutions will be realised in the external world via the GDA's effector(s).

These three computational processes form a recursive loop. Every new generation, modification and demolishment of the design in the external world will trigger a new round of interpretation, hypothesising and designing within the GDA. In this manner, the 3D virtual worlds are dynamically designed as needed.

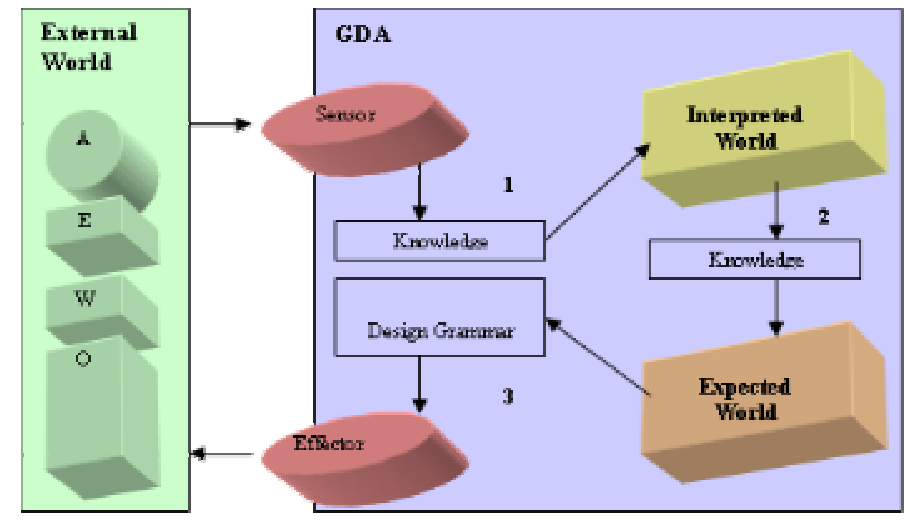

Figure 3 Three Major Computational Processes of the GDA Model: Interpretation (1), Hypothesising (2) and Designing (3)

\section{GENERATIVE DESIGN GRAMMAR}

The GDA's designing process requires a generative mechanism to produce design solutions for satisfying the design goals. Shape grammars (Stiny and Gips 1972) as design formalism and their applications have been developed in many design domains for over three decades. The core of the GDA's design component is a generative design grammar. The spatial components of this grammar are developed based on the notions of shape grammars.

Knight (2000) summarises the definition and the components of a shape grammar: a shape grammar is a set of shape rules that apply in a step-by-step manner to generate a set, or language of designs. A shape grammar is both descriptive and generative. The shape rules are the description of the spatial forms of the designs. They can also relate to the goals of a design project that may describe anything from functions to meanings to aesthetics and so on. The basic components of the shape rules are shapes. Shapes can be understood as points, lines, planes or volumes. The designs are generated using shape operations. Generally, applications of shape grammars 


\section{Dynamic Designs of 3D Virtual Worlds Using Generative Design Agents}

have two purposes. Firstly, shape grammars can be used as design tools to define design languages and styles. Secondly, shape grammars as design analysis tools, can be used to analyse existing designs in order to better understand the designs.

\subsection{Generative Design Grammar Framework}

The view of 3D virtual worlds as functional places that support professional activities provides a common ground for designing these worlds. This common ground highlights two key issues: activities and metaphor. Firstly, 3D virtual worlds exist for certain purposes supporting various online activities. Secondly, 3D virtual worlds apply the metaphor of architecture. Based on this understanding, designing $3 \mathrm{D}$ virtual worlds can be divided into the following four phases:

- To layout places for designated activities: each place has a volume that corresponds to certain online activities.

- To configure each place: the place then is configured with certain infrastructure, which provides boundary and visual cues for supporting the designated activities.

- To specify navigation methods: navigation in 3D virtual worlds can be facilitated to consider the use of way finding aids and hyper-links among different virtual places.

- To specify interactions: in general this is a process of ascribing behaviours to selected 3D objects in the world, so that the occupants can interact with these objects and each other.

The four phases of designing 3D virtual worlds define the basic structure of a generative design grammar framework that consists of four sets of design rules: layout rules, infrastructure rules, navigation rules and interaction rules (Figure 4). Following the structure of the generative design grammar framework, styles of static virtual architecture can be considered in terms of visualisation (layout of places and visual forms of infrastructure), navigation methods and interactions. They are three inseparable parts for providing an integral experience of 3D virtual worlds. Detailed analysis of styles of 3D virtual worlds can be found in Gu and Maher (2004).

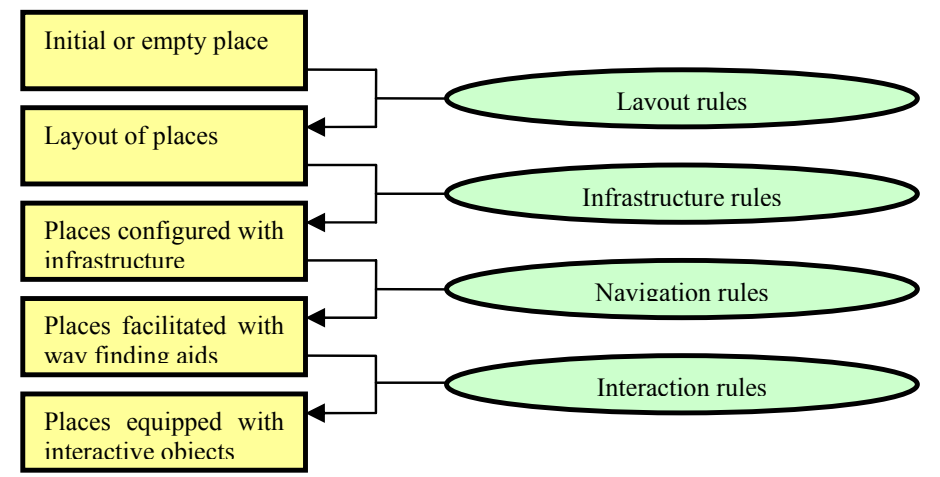

Figure 4 Structure of the Generative Design Grammar and the Firing Sequence of the Design Rules 
In shape grammars, state labels are usually applied to separate the shape rules of a grammar into different stages. The generative design grammar framework maintains this usage and further develops special sets of state labels to represent different design contexts. Each design rule is associated with a set of state labels, in order for a rule to be fired, the currently interpreted needs of the occupants and the state of the world need to match the design context represented by the state labels. In this manner, the application of the grammar is directed to generate designs that satisfy the needs of the occupants.

\subsubsection{Layout Rules}

Layout rules are spatial rules that produce layout of places according to different activities supported in a $3 \mathrm{D}$ virtual world. The use of different places for different activities creates a sense of movement for the avatars when changing activities. Because of the use of architectural metaphor, layout problems in 3D virtual worlds can have similar solutions as the ones in the built environments. However, unlike the built environments, 3D virtual worlds do not have to obey physical constraints. Many layout-related issues like adjacency do not need to strictly follow its physical counterpart, which offer designers more freedom in designing. Figure 5 shows two example layout rules that add different virtual gallery spaces to an existing reception space. The state labels of the rules are written above the LHS shape.

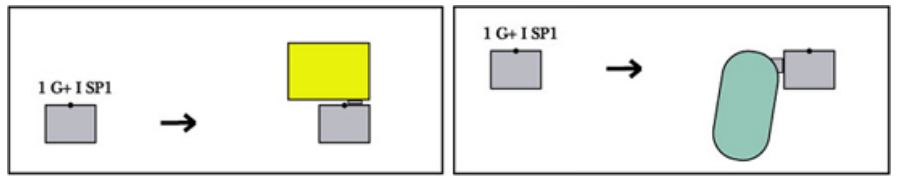

Figure 5 Two Example Layout Rules

\subsubsection{Infrastructure Rules}

Similarly, infrastructure rules are also spatial rules. They further configure each place by defining boundary and deciding objects placements, after the layout is produced. Like layout rules, there are many physical examples and principles to follow, but designers can also gain more freedom in designing as $3 \mathrm{D}$ virtual worlds are free from any physical constraints.

\subsubsection{Navigation Rules}

Navigation rules provide way finding aids such as visual cues or hyperlink portals, to support navigation in 3D virtual worlds. Way finding aids in virtual worlds has been studied with the direct reference to those in the built environments (Darken and Sibert, 1996; Vinson, 1999). There are at least two different types of way finding aids that can be integrated into 3D virtual worlds from the built environments. They are spatial elements and social elements. Spatial elements include paths, openings, 
hallways, stairs, intersections, landmarks, maps, signs and so on. Social elements refer to assistance from tour guides or other occupants.

Besides the above references from way finding aids in the built environments, virtual worlds have their unique forms of navigation as virtual places are hyperlinked. Most virtual worlds allow occupants to move directly between any two locations. The left diagram in figure 6 is an example navigation rule. The LHS of this rule shows that two virtual gallery spaces are separated in a virtual world, without direct access to each other. The RHS of this rule shows that a pair of hyperlinks is created inside these two galleries, which allows visitors to travel back and forwards freely. The right diagram in figure 6 shows the effect of this example navigation rule. The LHS is the interior of one of the virtual gallery spaces. The RHS shows that a hyper-link is created and appears as a colour stone on the floor, which will take the visitor directly to the other gallery space when it is stepped on.

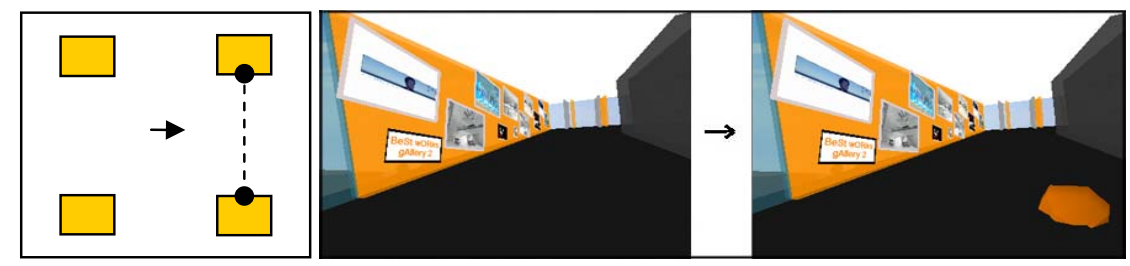

Figure 6 Left: an Example Navigation Rule; Right: the Effect of the Example Navigation Rule

\subsubsection{Interaction Rules}

Interaction rules ascribe appropriate behaviours to selected 3D objects in different virtual places. Therefore, occupants can interact with these $3 \mathrm{D}$ objects by activating their behaviours. In the built environments, the behaviours of a design artefact are causually related to its structure. This is not the case in $3 \mathrm{D}$ virtual worlds. Designers ascribe behaviours freely to the 3D objects. Figure 7 shows the effect of an example interaction rule. The LHS is an empty advertisement board outside a virtual building (at the top right corner of the image). The RHS shows the same advertisement board displaying a digital image in an animated sequence, after the interaction rule is fired, which reconfigures the properties of the board using a scripting language to enable the animation to be shown.



Figure 7 The Effect of an Example Interaction Rule 


\subsection{Generative Design Grammar Application}

The four sets of design rules are fired following the order of layout rules, infrastructure rules, navigation rules and interaction rules. By altering the sequence of the design rule application, different designs can be obtained. In this case, the occupants' needs of the moment and the current state of the virtual world are sensed and interpreted by the GDA, to be used to match the state labels, for directing the design grammar application. Therefore, 3D virtual places are generated differently during its use, in order to satisfy the changing needs of the occupants. Figure 8 illustrates a dynamic virtual gallery that changes overtime (from left to right) during its use in one particular session, to suit the needs of different moments.

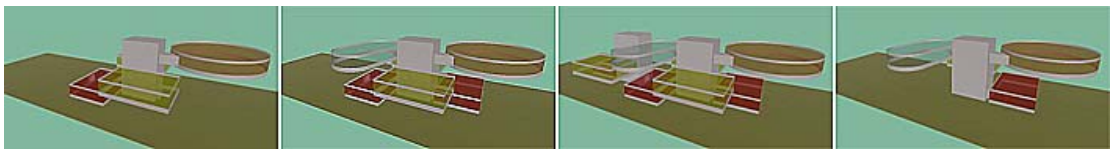

Figure 8 Example: A Dynamic Virtual Gallery Changes over Time

In the cases when there are more than one design rule that meet the current design context, a control mechanism is needed to resolve the conflict. In general, there are three main methods for controlling the generative design grammar application. They are random selection, human designer interference and evaluation-learning mechanism. The random selection method allow the system to randomly select one design rule from the set of rules that meet the current design context, for firing. The human designer interference method allows the system to turn to human designers or users for instructions once such a conflict occurs. The evaluation-learning mechanism provide a more dynamic but more complicated approach to allow the system to resolve the conflict based on past design experience and feedback. The design example shown in figure 8 uses the human designer inference method.

\section{CONCLUSION}

The generic GDA model presented in this paper enables 3D virtual worlds to be dynamically designed as needed. As the core of a GDA's design component, the generative design grammar includes the representation of design context of 3D virtual worlds in the forms of state labels, which can be used to match against the GDA's current interpretation for directing the grammar application. Highlighted with the GDA's generative capabilities, this research provides new insights for 3D virtual worlds from the following perspectives:

- The GDA model introduces dynamics and autonomy to the designs of 3D virtual worlds. Virtual worlds designed with the GDA model do not have a static infrastructure like the built environments. It is designed for a particular "moment", and reflects its inhabitants' interests of that "moment".

- The generative design grammar framework serves as a base for developing generative design grammars with different styles that suits different purposes. 


\section{Dynamic Designs of 3D Virtual Worlds Using Generative Design Agents}

- The generative design grammar framework also provides a foundation to formally study the styles of $3 \mathrm{D}$ virtual worlds. Compared to other novice designs, virtual worlds designed with a specific style in mind will achieve better consistency in terms of visualisation, navigation and interaction, and this consistency provides a strong base to assist its occupants' orientations and interactions in the virtual worlds.

The future extension of this research will be focused on agent communication. The first direction is to study the GDA's interpretation process in a complex multi-GDA 3D virtual world. The second direction is to study the collective design styles of 3D virtual worlds shared by a society of GDAs.

\section{REFERENCES}

Darken, Rudolf P., and John L. Sibert. 1996. Way Finding Strategies and Behaviours in Large Virtual Worlds. In Proceedings of ACM SIGCHI'96: 142-149. New York: ACM.

Gero, John S. 2002. Computational Models of Creative Designing Based on Situated Cognition. In Proceedings of Creativity and Cognition 2002, ed. Tom Hewett and Terence Kavanagh: 3-10. New York: ACM.

Gu, Ning, and Mary L. Maher. 2004. Generating Virtual Architecture with Style. In Proceedings of ANZAScA 2004, ed. Zbigniew Bromberek: 141-147. University of Tasmania.

Huhns, Michael N., and Larry M. Stephen. 1999. Multiagent Systems and Society of Agents. In Multiagent Systems: A Modern Approach to Distributed Artificial Intelligence, ed. Gerhard Weiss: 79-120. Cambridge: MIT Press.

Knight, Terry W. 2000. Shape Grammars in Education and Practice: History and Prospects. International Journal of Design Computing 2. Available from http://www.arch.usyd.edu.au/kcde/journal/vol2; accessed 24 January 2005.

Lakoff, George, and Mark Johnson. 1980. Metaphors We Live by. Chicago: University of Chicago Press.

Maher, Mary L., and John S. Gero. 2002. Agent Models of Virtual Worlds. In Proceedings of ACADIA 2002: 127-138. California State Polytechnic University.

Maher, Mary L., P-S Liew, Ning Gu, and Lan Ding. 2003. An Agent Approach to Supporting Collaborative Design in 3D Virtual Worlds. In Proceeding of eCAADe 2003, ed. Wolfgang Dokonal and Urs Hirschberg: 47-52. Graz University of Technology.

Russell, Stuart, and Peter Norvig. 1995. Artificial Intelligence: A Modern Approach. Englewood Cliffs: Prentice Hall.

Stiny, George, and James Gips. 1972. Shape Grammars and the Generative Specification of Painting and Sculpture. In Proceedings of Information Processing 71, ed. C.V. Freiman: 1460-1465. Amsterdam: North Holland.

Vinson, Norman G. 1999. Design Guidelines for Landmarks to Support Navigation in Virtual Environments. In Proceedings of CHI'99: 278-285. Pittsburgh.

Wooldridge, Michael, and Nicholas R. Jennings. 1995. Intelligent Agents: Theory and Practice. Knowledge Engineering Review 10(2): 115-152. 